\title{
ONE-BATH ONE-STEP DYEING OF POLYESTER/COTTON (PC) BLENDS FABRIC WITH DISPERSE DYES AFTER ACETYLATION OF COTTON
}

\author{
${ }^{1}$ Girmaye Kumsa, ${ }^{2 *}$ Gemeda Gebino and ${ }^{1}$ Gezu Ketema \\ ${ }^{1}$ Department of Textile Chemistry, College of Engineering and Technology, Wolkite University, Ethiopia \\ ${ }^{2}$ Textile Chemistry in Ethiopian Institute of Textile and Fashion Technology, Bahir Dar University, Ethiopia \\ * Correspondence: gemedalvgebino@gmail.com
}

\begin{abstract}
Usually, the two-bath dyeing process using disperse dyes and reactive dyes separately was employed for the dyeing of PC blends. The cost of the double bath, dyeing cycle, energy consumption and chemical consumption is quite higher than the one-step or single bath dyeing methods. In this study, the one-bath dyeing process using one kind of dye was investigated. Polyester cotton blends dyed in one-bath one-step dyeing methods with disperse dye after surface modifications of cotton by acetylation methods were studied. Surface modification of cotton was carried out using fibrous acetylation methods. The effect of acetic anhydride and time on percent acetyl content at room temperature was studied. Modified polyester/cotton was carried out in HTHP dyeing machine incorporates with different dye concentrations and dyeing temperature. Surface chemistry, thermal decomposition property and moisture regain of modified polyester/ cotton blend are evaluated. The color strength of the dyed fabrics and their fastness properties to washing, light, and rubbing as well as tear strength and abrasion resistance were evaluated. The effect of dye concentration and temperature color strength, tensile strength warp and weft direction was assessed. The optimum value for surface modification was obtained with a concentration of acetylation agent $16 \%$ and time of reaction 2.5 hours, gave percent acetylation of 34. An FTIR spectrum shows acetylation resonance. The experiment result of dyeing showed that the optimum value was obtained with dye concentration above $1 \%$ at a temperature of $120^{\circ} \mathrm{C}$, warp tensile strength decreased by $12 \%$ and weft tensile strength was decreased by $9 \%$ from the control half-bleached fabric. Results of this study showed that one-step one-bath dyed modified polyester/cotton blend with disperse dye fabric presents good fastness property compared with conventional two-bath dyed fabric as well as color strength values.
\end{abstract}

Keywords: Polyester/cotton blend, One-bath one-step dyeing, surface modification, Acetylation, Disperse dye. 


\section{INTRODUCTION}

Polyester/cotton fabrics are widely used in the clothing industry and other application areas due to their complementary properties $[\underline{1}, \underline{2}]$. Polyester fibers provide crease recovery, dimensional stability, abrasion resistance, tensile and easy-care properties, while cotton fibers give moisture absorption and anti-static characteristics and minimize pilling rolls. This make polyester/cotton blends are famous due to their aesthetic value, user-friendly performance and limitations of both fibers are balanced adequately by blending these two fibers making perfect blend [3].

Dyeing of polyester/cotton blends poses some challenges to dyer as polyester shows a hydrophobic character while cotton shows a hydrophilic character making it inevitable to dye them with chemically different class of dyes []].

Two-bath or one-bath-two-step dyeing method employing proper dyes and chemicals for each fiber usually carry out a blend dyeing of polyester/cotton $[\underline{5}, \underline{6}]$. However, these dyeing methods are relatively long and complicated. The process of one-bath-two-step dyeing process is shorter than the two-bath method but its drawback is lower dye ability from migration and poor reproducibility since two different kinds of dyes used in the same bath [7]. The one-bath one-step dyeing process of polyester/cotton blends with disperse/ reactive dyes has the advantages over the conventional dyeing processes on reducing the dyeing cycle as well as energy consumption [8].

Surface modification of the polymeric fibers without changing the bulk properties has been a classical research topic. Surface modification of textile fiber enhance and increase their dye ability, because of many functional groups bond to the surface such as hydroxyl, ester, carboxyl, and carbonate groups, instead of one desired functional group [ $\underline{9}, \underline{10}]$. Chemically modifying of cellulose fibers at the surface without appreciably changing their native structure crystalline and morphology achieved by esterification reactions changing the surface properties through modification of fiber surface chemistry except to decrease the natural hydrophilic character of cellulose fibers [11].

Acetylation is an esterification reaction, which plasticizes natural fibers by-introducing acetyl functional groups $(\mathrm{CH} 3 \mathrm{CO}-)[\underline{12}, \underline{13}]$. In this reaction, acetic acid $(\mathrm{CH} 3 \mathrm{COOH})$ is produced as a by-product that must be separated from the reaction mixture to prevent damage to the fibers. When treated with acetic anhydride $\left(\mathrm{CH}_{3}-\mathrm{C}(=\mathrm{O})-\mathrm{O}-\mathrm{C}(=\mathrm{O})-\mathrm{CH}_{3}\right)$, it replaces the hydroxyl 
groups present in the cell wall polymers with acetyl groups, making the fibers hydrophobic in nature $[\underline{14}, \underline{15}]$.

This work involves one-bath one-step dyeing of polyester/cotton (PC) blends with disperse dye after acetylation of cotton to reducing the dyeing cycle as well as energy consumption. Development of these dyeing methods suitable to PC blends used for cold weather wearing cloth, especially for technical textiles and home furniture since, functional and performance property of this fabric needed rather than its comfort property for this specific application area.

\section{MATERIALS AND METHODS}

\subsection{Materials and Reagents}

Half bleach polyester/cotton (PC) fabrics with a blend ratio of 65:35 samples at the specification of 90 picks per inch and 152 ends per inch and 201 GSM (gram per meter square). The chemicals used were obtained from Almeda Textile PLC and EiTEX laboratory respectively. Acetic acid, acetic anhydride, zinc chloride, C.I. Disperse dye yellow-S-6GF200 and reactive dye yellow S-8G was purchased from chemo scientific trading in Addis Ababa Ethiopia. These chemicals are directly used without further purification.

\subsection{Methods}

\subsubsection{Acetylation process}

Conventionally, acetylation is carried out by the reaction of glacial acetic acid as a solvent with acetic anhydride as the acetylating agent in the presence of zinc chloride as a catalyst. Acetylation using zinc chloride as a catalyst causes a reduction in fabric strength compared to using sulphuric acid according to [16]. The reaction conditions depend on several factors including time of reaction and concentration of acetic anhydride that keeps varying to determine the optimum conditions for the acetylation. The concentration of acetic anhydride used for acetylation were 10 to $20 \%$ and $6 \mathrm{gpl}$ of zinc chloride as a catalyst at MLR of 1:10 and the time of reaction was $1-4 \mathrm{hr}$ at room temperature due to temperature effects tensile strength of the fabric $[16, \underline{17}]$. The combination of glacial acetic acid at 1:2 ratios to sample weight used for acetylation method. The acetylation was done using a central composite design in 13 runs. 


\subsubsection{Percent Acetylation}

Percent acetylation is the percentage of the acetyl group that has replaced the hydroxyl group in the pretreated sample using titration [18]. The estimation of acetyl content was carried out, using an aqueous sodium hydroxide solution to hydrolyze the cellulose acetate and back estimating the excess sodium hydroxide with a standard hydrochloric acid solution.

Two grams of the sample taken and then exactly $40 \mathrm{ml}$ of 0.5 normality of sodium hydroxide solution was added into the flask to hydrolyze the acetylated cotton and warmed at $45^{\circ}$ for 15 minutes. Afterward, the flask was kept for 24 hours to complete hydrolysis at room temperature. Then excess sodium hydroxide was back titrated against 0.5 normality hydrochloric acid solution using phenolphthalein as an indicator.

$$
\text { Acetylation percentage }=\frac{(\mathrm{B}, \mathrm{cm} 3)-(\mathrm{S}, \mathrm{cm} 3) \times \mathrm{N} \times 4.3}{\mathrm{~W}} * 100 \% \ldots \ldots \ldots . .1
$$

Where, $\mathrm{B}=\mathrm{ml}$ of 0.5 normality of hydrochloric acid used to titrate blank

- $\mathrm{S}=\mathrm{ml}$ of 0.5 normality of hydrochloric acid used to titrated sample

$\circ \mathrm{N}=$ is 0.5 , the normality of hydrochloric acid and sodium hydroxide used for the titration.

○ $4.3=$ is related to the molecular weight of the acetyl group $\left(\mathrm{CH}_{3} \mathrm{CO}\right)$.

$\circ \mathrm{W}=$ weight of sample in gr.

\subsubsection{Dyeing method}

Dyeing methods carried out after modification of cotton part with disperse dye in one bath using high temperature and high-pressure (HTHP) machine at different dyeing temperatures to find optimum dyeing temperature of this blend with minimum loss of tensile strength of cotton portion. The PC blend was subjected to dyeing using both the conventional way of dyeing and one- bath one-step dyeing method. Dyeing was carried out with disperse dye, after modification of cotton part dyed in HTHP dyeing machine at MLR 1:20. $\mathrm{pH}$ was maintained at 5-6 using acetic acid. The dye bath temperature was varying from $100-140^{\circ} \mathrm{C}$ for $60 \mathrm{~min}$. The dyeing was rinsed and then reduction cleared in an aqueous solution of $2 \mathrm{gpl}$ sodium hydroxide $(\mathrm{NaOH})$ and $2 \mathrm{gpl}$ sodium hydrosulphite $\left(\mathrm{Na}_{2} \mathrm{~S}_{2} \mathrm{O}_{4}\right)$ at $60^{\circ} \mathrm{C}$ for 15 minutes.

When dyeing PC blend modified with disperse dye, Design Experiment 11 with 29 number of runs were used to determine the effect of dye concentration and temperature to find best dyeing temperature at a minimum loss of strength along warp and weft direction. 


\subsection{Characterization of Modified PC Blends}

\subsubsection{Instrumentation}

All the acetylation reactions of the cotton cellulose were carried out in a 500ml laboratory beaker in a Crest Ultrasonic cleaner (Model No. P230 with 115volt). The surface chemistries of untreated and acetylated cotton fabrics were evaluated by using Fourier Transform Infrared spectroscopy (Perkin Elmer FTIR) according to ASTM 7575 test standard methods. The results were recorded in the frequency range of $4000-400 \mathrm{~cm}^{-1}$ by using 20 scans. Thermal analysis was performed using TGA (Perkin Elmer) instrument (Model No. TGA 4000) to distinguish thermal stability of blend fabric before and after modification. Measurements were conducted from ambient temperature to $500^{\circ} \mathrm{C}$ at a heating rate of $20^{\circ} \mathrm{C} / \mathrm{min}$ in a nitrogen atmosphere. The result of TGA was used to study the denaturation properties of modified and unmodified polyester/cotton blends. Tensile strength test was done according to the ASTM D-5034-textile grab test method using Tensolab 100 (Mesdan Lab, Italy). The breaking force and elongation were recorded.

\subsubsection{Measurement of moisture regains}

Moisture regains of the modified PC blended fabrics were determined as per the ASTM standard test method 2654. The modified blended fabrics were conditioned in a standard testing atmosphere of $20^{\circ} \mathrm{C}$ and $65 \%$ relative humidity for $24 \mathrm{~h}$ before actual testing (Equation 2).

$$
\operatorname{Regain}(\%)=\frac{(\% a) R a+(\% b) R b}{\% a+\% b} \ldots \ldots \ldots \ldots . . . .2
$$

Where: $\% \mathrm{a}=$ proportion of fiber ' $\mathrm{a}$ ', $\%$; and $\% \mathrm{~b}=$ proportion of fiber ' $\mathrm{b}$ ', $\%$

\subsubsection{Color Strength Measurements}

The color strength of dyed fabric samples was measured using a spectrophotometer (Gretag Macbeth color eye 3100). The test is done by the procedure set by the AATCC 6-2003 test standard methods. The color strength of dyed fabric samples was measured from the reflectance value at a maximum wavelength from which surface color strength using $\mathrm{K} / \mathrm{S}$ value. . K/S is a function of color depth and is represented by the equation of Kubelka and Munk (Equation 3).

$$
\frac{K}{S}=\frac{\left(1-r R^{2}\right.}{2 R} \ldots \ldots \ldots \ldots \ldots \ldots . \cdots \cdots
$$


Where $\mathrm{R}$ is the reflectance of the dyed fabric; $\mathrm{K}$ is the sorption coefficient, and $\mathrm{S}$ is the scattering coefficient. The spectrophotometer was standardized for a 1-inch diameter specimen viewing aperture in reflectance - specular included mode. Illuminant D65 and the CIE 10-degree observer were used.

\subsubsection{Fastness Properties}

Dyed modified PC blended fabric was tested for colorfastness to rubbing according to AATCC standard test method 116-2001 using Crock-meter (Mesdan Lab, Italy). Colorfastness to washing was done according to the AATCC standard test method 61-2001 using Laundry-O-Meter (Mesdan Lab, Italy). Colorfastness to light was determined according to ISO105-B02 standard test method using SOLAR BOX (Italy), 2013. Colorfastness to perspiration was determined according to AATCC standard test method 15-1997 (AATCC, 2001). Change in the shade was evaluated using the AATCC Gray Scale for Evaluating Change in Color and color transfer was evaluated using the AATCC Gray Scale for Evaluating Staining (AATCC, 2001). Finally, the crease recovery was determined according to AATCC-128 standard test methods using Shirley crease recovery tester.

\section{RESULTS AND DISCUSSION}

\subsection{Optimization of the Acetylation}

In this study, the acetylation process was made for surface modification of cotton parts in PC blends. Different parameters are considered during the acetylation process such as concentration, time and percent of acetylation (Table 1). The response percent acetylation used to evaluate the degree of the acetyl group that has been substituted in place of the hydroxyl group of cotton cellulose. The experimental design was used to study the relationship between the variables acetic anhydride concentration, and reaction time at room temperature. Analysis of variance ANOVA determines whether the variables acetic anhydride concentration and time are significantly affecting the percent acetylation.

Table 1. Acetylation process

\begin{tabular}{|l|l|l|l|l|}
\hline & & Factor 1 & Factor 2 & Response 1 \\
\hline Std unit & Run & A: concentration (\%) & B:time (hr) & Percent acetylation (\%) \\
\hline 3 & 1 & 10 & 4 & 22 \\
\hline 2 & 2 & 20 & 1 & 19 \\
\hline 4 & 3 & 20 & 4 & 27 \\
\hline
\end{tabular}




\begin{tabular}{|l|l|c|c|c|}
\hline 7 & 4 & 15 & 0.5 & 15 \\
\hline 9 & 5 & 15 & 2.5 & 34 \\
\hline 1 & 6 & 10 & 1 & 16 \\
\hline 12 & 7 & 15 & 2.5 & 35 \\
\hline 10 & 8 & 15 & 2.5 & 32 \\
\hline 8 & 9 & 15 & 4.6 & 29 \\
\hline 11 & 10 & 15 & 2.5 & 32 \\
\hline 5 & 11 & 8 & 2.5 & 24 \\
\hline 6 & 12 & 22 & 2.5 & 26 \\
\hline 13 & 13 & 15 & 2.5 & 31 \\
\hline
\end{tabular}

Figure 1 shows the plots of the normality test of the residuals for the degree of substitution, warp way tensile strength and weft way tensile strength respectively. It is observed that in all the graphs, the residuals lie on the straight line, which indicates that the distribution of residuals for percent acetylation is normal. From the results, the assumption of constant variance is confirmed and the suggested model is suitable.

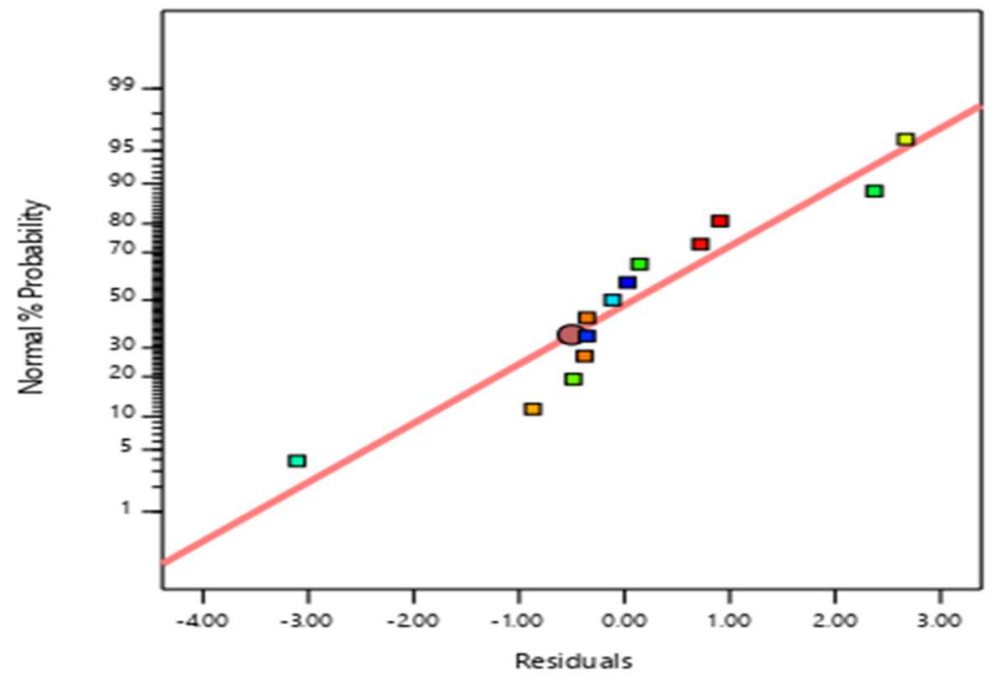

Figure 1 . Normality \% probability vs residual plot for percent acetylation

Table 2. ANOVA analysis of acetylation condition

\begin{tabular}{|l|r|r|r|r|r|l|}
\hline \multicolumn{1}{|c|}{ Source } & Sum of Squares & df & Mean Square & F-value & p-value & \\
\hline Model & 509.00 & 4 & 127.25 & 33.67 & $<0.0001$ & significant \\
\hline A-con & 16.53 & 1 & 16.53 & 4.37 & 0.0099 & \\
\hline B-time & 132.17 & 1 & 132.17 & 34.97 & 0.0004 & \\
\hline $\mathrm{A}^{2}$ & 155.34 & 1 & 155.34 & 41.10 & 0.0002 & \\
\hline $\mathrm{B}^{2}$ & 250.27 & 1 & 250.27 & 66.21 & $<0.0001$ & \\
\hline
\end{tabular}




\begin{tabular}{|l|r|r|r|r|r|l|}
\hline Residual & 30.24 & 8 & 3.78 & & & \\
\hline Lack of Fit & 22.75 & 4 & 5.69 & 3.04 & 0.1536 & not significant \\
\hline Pure Error & 7.49 & 4 & 1.87 & & & \\
\hline Cor Total & 539.24 & 12 & & & & \\
\hline
\end{tabular}

Table 2 shows P-values less than 0.0500 and F-value of 33.67 implies the model is significant. There is only a $0.01 \%$ chance that an F-value this large could occur due to noise. The concentration of acetic anhydride (A and $\mathrm{A}^{2}$ ) and $\mathrm{B}^{2}$ (square of time) are significant model terms. Values greater than 0.1000 indicate the model terms are not significant. The Lack of Fit F-value of 3.04 implies there is a $15.36 \%$ chance that a Lack of Fit F-value could occur due to noise. Therefore, this ANOVA table indicates the process is significant and the model is fit.

\subsubsection{Effect of acetic anydride concentration on percent acetylation}

Glacial acetic acid is the solvent, which functions as the swelling agent for the cotton by increasing the accessibility of the cotton hydroxyl groups to the acetylating agent (acetic anhydride) [18, 19]. It was proposed that at the beginning of the reaction, the reaction was heterogeneous, and $\mathrm{OH}$ groups on the surface of solid cellulose will be preferentially acetylated and then, as the acetylation progressed, the acetylation of cellulose gradually continued with dissolved in the reaction medium. The extent of esterification was found to increase with the increase in esterifying agents. Maximum esterification of $35 \%$ was obtained when the amount of acetic anhydride was increased from $7 \%$ to $15 \%$. Further increasing the concentration of acetic anhydride from $15 \%$ to $22 \%$ shows a decrease in acetylation percentage (Table 1). A high concentration of acetic anhydride reduce percent of acetyl content, acid-catalyzed hydrolysis of the ester is responsible for the reduction of percent acetylation and water molecules will react with acetic anhydride beyond $15 \%$ of acetic anhydrides and a high concentration of acetic anhydride can cause degradation of carbohydrates which results in a decrease in weight percentage gain. At the fibrous stage, without destroying fibrous structure the maximum percent acetyaltion is $32-35 \%$ [20]. 


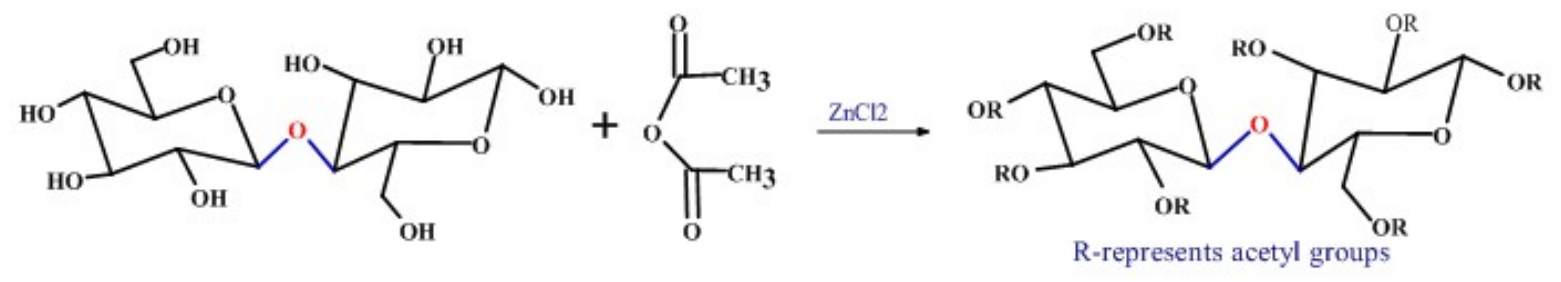

Figure 2. Acetylation reaction mechanism of cellulose

\subsubsection{Effect of time on percent acetylation}

The extent of values of percent acetylation was found to increase with prolonging the reaction period from 1 to 4.62 hours for all the experiments performed. Further increase in the reaction time from 2.5 to 4.5 hours, the percent acetylation was found to decrease from $35 \%$ to $32 \%$ (Table 1). The maximum value of acetate formation was observed to be $35 \%$ at a reaction time of 2.5 hours. Longer reaction times result in the hydrolysis of the ester groups or cellulose acetate which causes a reduction of percent acetylation. Figure 3 shows the effect of reaction time on acetate formation. It can be concluded from the time variation study that a maximum level of

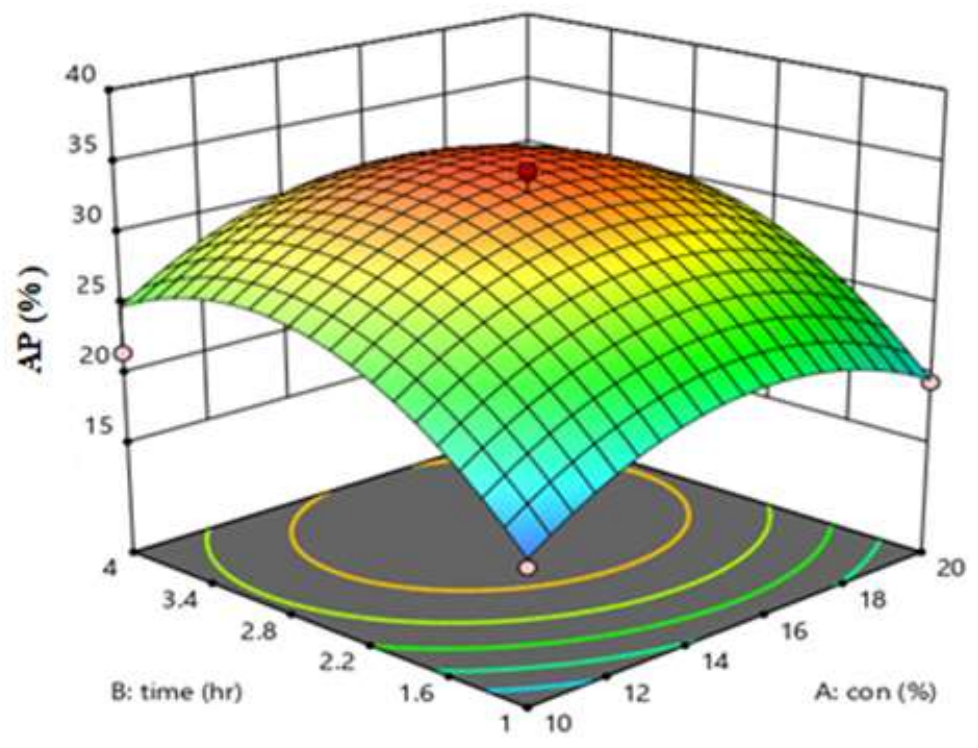

Figure 3: Effect of acetic anhydride concentration and time on percent acetyl content.

acetylation was reached by continuing the reaction for the optimum time period under the set of reaction conditions. After acquiring the optimum extent of esterification, the cellulose molecule did not undergo further electrophilic displacement with the acylpyridinium complex. Thus the 
leveling-off in the esterification value may be viewed as the establishment of equilibrium between the products and the reactants under the chosen set of reaction conditions. The maximum values were achieved for the percent acetylation after the optimum time (2.5hours) duration and at $16 \%$ concentration were $34 \%$.

\subsection{FTIR analysis}

Since the properties of cotton cellulose acetylated are one of the significant factors contributing to the dyeability of the material, differences between the cotton-acetylated sample and cotton cellulose were evaluated to understand the chemical change that has taken place in acetylation (Figure 4). Acetylated cotton characterized by FTIR to ensure the reaction of hydroxyl groups with acetic anhydride. A typical loss in transmittance band for the acetylated cotton is observed in the range $3600 \mathrm{~cm}-1$ to $3300 \mathrm{~cm}-1$ that is assigned to the $\mathrm{CH}_{3} \mathrm{CO}^{-}$of the acetyl group as also reported by [21]. The stretch is not as broad or strong as it appeared in the cotton $\mathrm{OH}$-stretch in this region. The transmittance band from $3400 \mathrm{~cm}^{-1}$ to $3000 \mathrm{~cm}^{-1}$ is very broad in the untreated cotton which signifies the large numbers of $-\mathrm{OH}$ groups in the cotton [22]. The intensity of the $\mathrm{OH}$ absorption band that the hydroxyl group contents in the cotton fabric were reduced after the reaction. The ester carbonyl absorption peaks at $1712 \mathrm{~cm}^{-1}$ and the $\mathrm{C}-\mathrm{H}$ stretch at $1242 \mathrm{~cm}-1$ to form an acetyl group [23]. The strong sharp peak at $1250 \mathrm{~cm}^{-1}$ and $1300 \mathrm{~cm}^{-1}$ may be due to the $\mathrm{C}-\mathrm{O}$ stretching of the polymer backbone. The observed strong sharp peaks were due to the cellulosic component of the fiber materials.

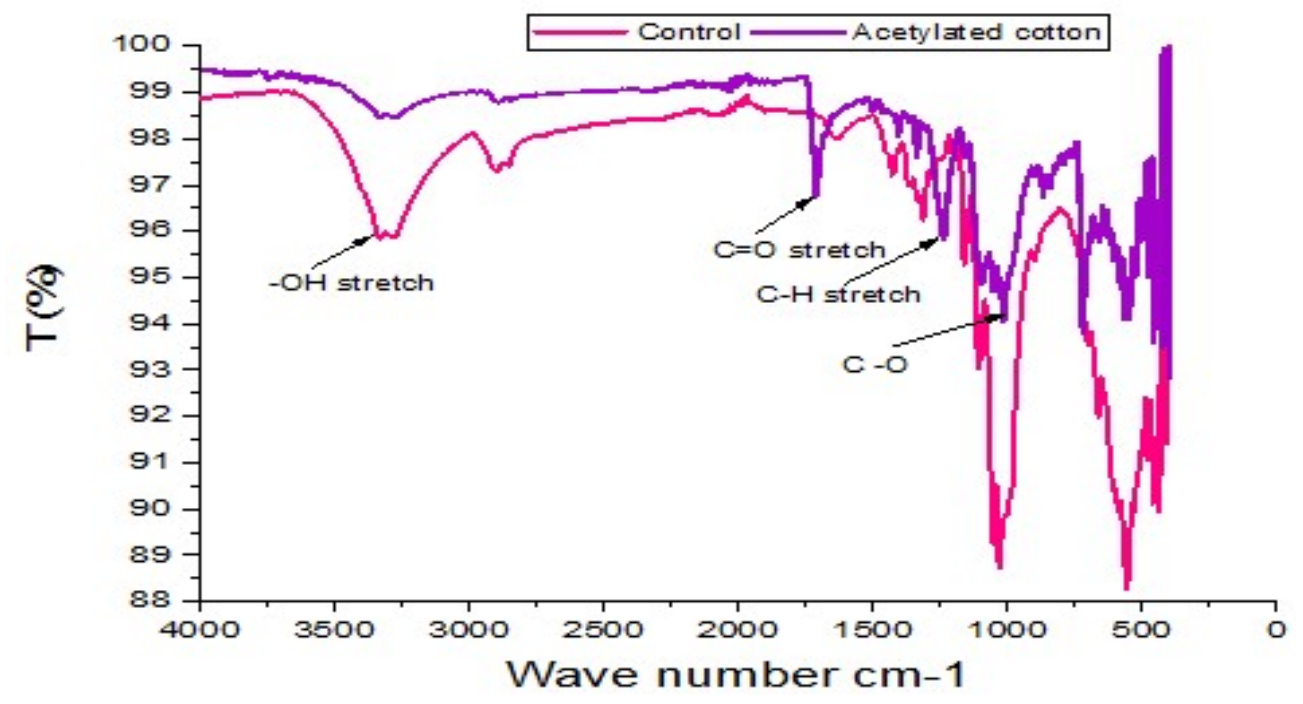

Figure 4. FTIR Spectra of control or cotton fabric and acetylated cotton fabric. 


\subsection{Moisture regain of modified PC blend}

Moisture regains and content of PC blend fabric were calculated by equation 2 [24]. As Figure 5 shows the average test result of four pre-treated polyester/cotton blended samples moisture regain and contents are $2.176 \%$ and $1.033 \%$ respectively. From the result obtained, moisture of PC blends after acetylation are reduced due to the hydrophilic group of cotton cellulose are replaced by acetyl group with a medium degree of substitution. Since cellulose fibres or fabric can be directly acetylated without destroying their fibrous structure [25].

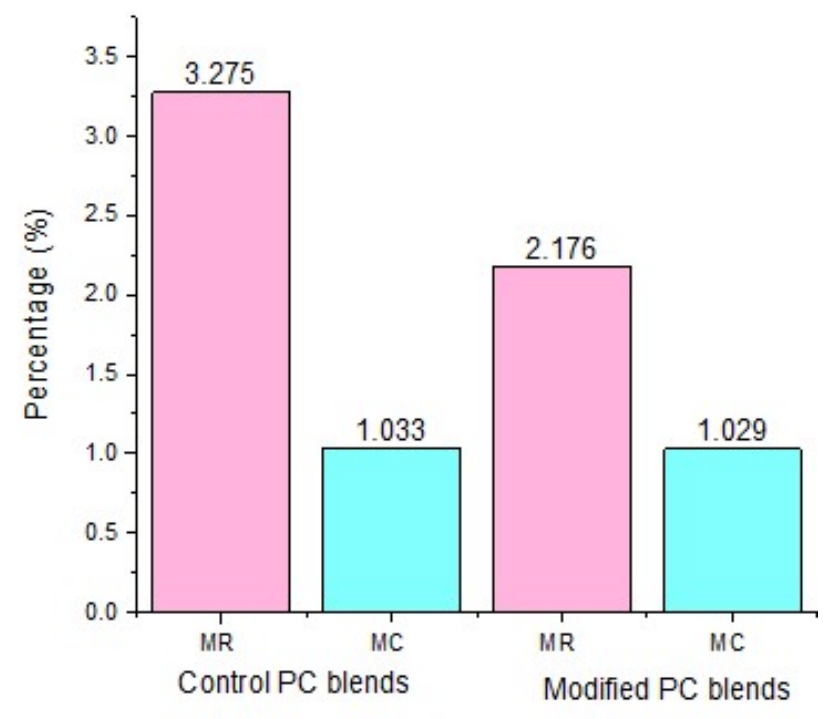

Figure 5. Comparison of moisture regain and contents of control and modified PC blends

\subsection{Thermogravimeteric Analysis}

The TG curves of the PC blends and surface modified cotton fabrics are shown in (Figure 6). The TG curves were separated into three sections. At first, the weight loss in the temperature range of $100{ }^{\circ} \mathrm{C}$ to $150{ }^{\circ} \mathrm{C}$ could be assigned to the evaporation of water. The weight loss is a direct measure of the volatiles formed during decomposition, and the rate of weight loss is an indication of the thermal decomposition kinetics. In the range of $250{ }^{\circ} \mathrm{C}$ to $300{ }^{\circ} \mathrm{C}$, there appeared a minor thermal decomposition of residues. The result of TG curve showns that all the major decompositions are located in the range of $350^{\circ} \mathrm{C}$ to $500^{\circ} \mathrm{C}, 354^{\circ} \mathrm{C}$ to $554^{\circ} \mathrm{C}$ for control $\mathrm{PC}$ blends and modified polyester/cotton blends respectively. Maximum thermal decompostion of control and modified PC blends are $714.87^{\circ} \mathrm{C}$ and $718.12^{\circ} \mathrm{C}$ as well as the rate of weight loss at this temperature is $92.75 \%$ and $88.99 \%$ respectively from TGA data. The residual mass percentage beyond this temperature corresponds to the combined ash and fixed carbon 
percentages. When comparing the samples, it was discovered that the major decomposition temperature of modified PC blend fabric was higher than normal fabric. The result suggests that the increase of thermoplasticity and heat resistance property of cotton was caused by the acetylation [26]. This icrement of thermal resistance required in use for technical textiles and home furniture application area of this blends fabric.

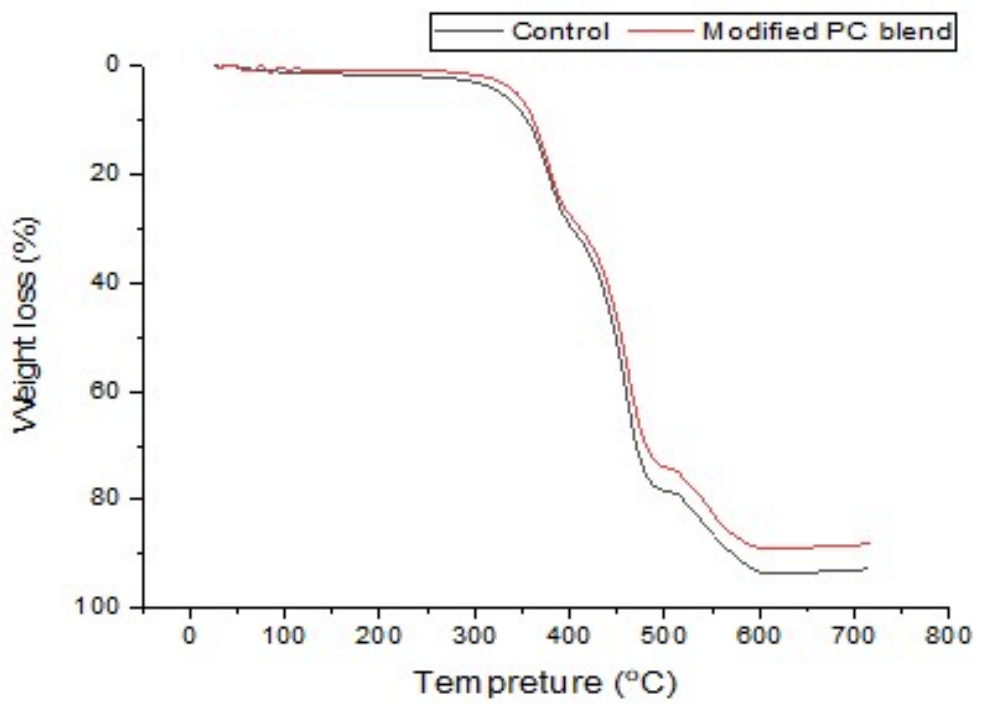

Figure 6. TG curve of polyester/cotton blends and modified polyester/cotton blends

\subsection{Crease Recovery of Modified PC Blends}

Crease recovery angle of control and modified PC blends after five-minute load application was assessed. The crease recovery angles obtained in warp directions were higher than the crease recovery angles obtained in weft directions for both control and modified PC blends. The crease recovery of control samples is less than modified samples in both warp $\left(52^{\circ}\right.$ and $\left.57^{\circ}\right)$ and weft $\left(37^{\circ}\right.$ and $\left.42^{\circ}\right)$ direction respectively as shown in Figure 7 . Because the esterification process improves anti-pilling and wrinkle recovery properties of cotton fabrics due to hydroxyl groups of cellulose are replaced by flexible acetyl group through an esterification reaction. The crease action applied on the fabric was shared by a higher number of yarns in warp direction which resulted in a better crease recovery. PC blend fabric was pretreated and acetylation is an esterification reaction, which plasticizes cotton by introducing acetyl functional groups. These results provide a tool for the occurrence of esterification reactions and also manifest the morphological changes incurred in the blend substrate which provides a better crease recovery angle. 


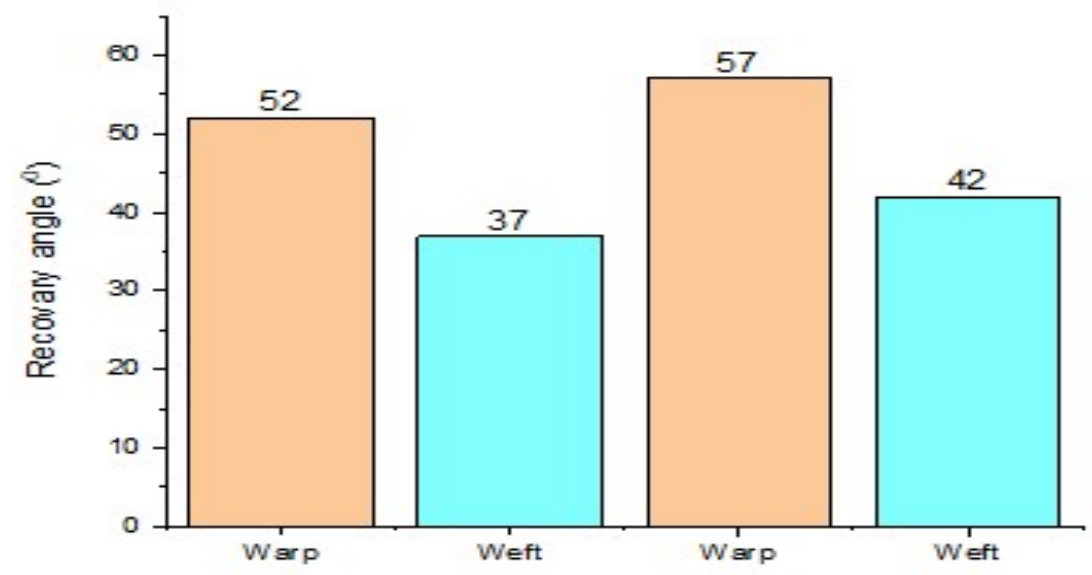

Control PC blends

M odified PC blends

Figure 7. Comparison of crease resistance of control and modified PC blends

\subsection{Dyeing of Modified PC Blends}

Table 3. Experimental result of dyeing process

\begin{tabular}{|c|c|c|c|c|c|}
\hline Run & $\begin{array}{c}\text { A: Concentration } \\
(\%)\end{array}$ & $\begin{array}{c}\text { Factor 2 } \\
\text { Temperature }\end{array}$ & Response 1 & Response 2 & Response 3 \\
\hline 1 & 3.4 & 120 & 5.2 & $\begin{array}{c}\text { Tensile strength } \\
\text { warp (N) }\end{array}$ & $\begin{array}{c}\text { Tensile strength } \\
\text { weft (N) }\end{array}$ \\
\hline 2 & 3 & 130 & 5.3 & 1130 & 617 \\
\hline 3 & 3.4 & 120 & 5.1 & 1128 & 605 \\
\hline 4 & 2 & 120 & 4.1 & 1140 & 620 \\
\hline 5 & 2 & 120 & 4.1 & 1125 & 618 \\
\hline 6 & 1 & 130 & 3.8 & 1100 & 623 \\
\hline 7 & 3 & 110 & 3.9 & 1162 & 611 \\
\hline 8 & 2 & 106 & 2.1 & 1190 & 642 \\
\hline 9 & 2 & 106 & 2.1 & 1195 & 664 \\
\hline 10 & 1 & 130 & 3.9 & 1090 & 657 \\
\hline 11 & 1 & 130 & 3.9 & 1080 & 607 \\
\hline 12 & 1 & 110 & 2.9 & 1170 & 645 \\
\hline 13 & 2 & 134 & 4.8 & 1015 & 601 \\
\hline 14 & 3 & 130 & 5.5 & 1096 & 605 \\
\hline 15 & 2 & 120 & 4.2 & 1120 & 614 \\
\hline 16 & 2 & 120 & 4.2 & 1132 & 625 \\
\hline 17 & 2 & 120 & 4.1 & 1125 & 619 \\
\hline 18 & 1 & 110 & 2.8 & 1150 & 638 \\
\hline 19 & 1 & 110 & 2.9 & 1170 & 644 \\
\hline 20 & 3 & 130 & 5.5 & 1102 & 609 \\
\hline 21 & 3.4 & 120 & 5.2 & 1136 & 1130 \\
\hline 22 & 0.6 & 120 & 3.1 & & 622 \\
\hline & & & & & \\
\hline
\end{tabular}




\begin{tabular}{|l|c|c|c|c|c|}
\hline 23 & 2 & 134 & 4.8 & 1030 & 590 \\
\hline 24 & 0.6 & 120 & 2.9 & 1140 & 618 \\
\hline 25 & 0.6 & 120 & 3.1 & 1139 & 621 \\
\hline 26 & 2 & 106 & 1.9 & 1200 & 662 \\
\hline 27 & 3 & 110 & 3.8 & 1155 & 640 \\
\hline 28 & 3 & 110 & 3.9 & 1153 & 642 \\
\hline 29 & 2 & 134 & 4.7 & 1022 & 596 \\
\hline
\end{tabular}

Analysis of variance (ANOVA) was used to determine whether the variables like dye concentration and temperature are significantly affecting the response values of color strength and tensile strength in warp and weft directions. Normal \% probability vs residual showed on Figure 8 for color strength and tensile strength in warp and weft direction lies on straight lines which indicates the distribution of residuals for all test results are normal and the assumption of constant variance is confirmed and suggests the model is suitable. The normal probability plot of the residuals is approximately linear supporting the condition that the error terms are normally distributed in both color strength and tensile strength.

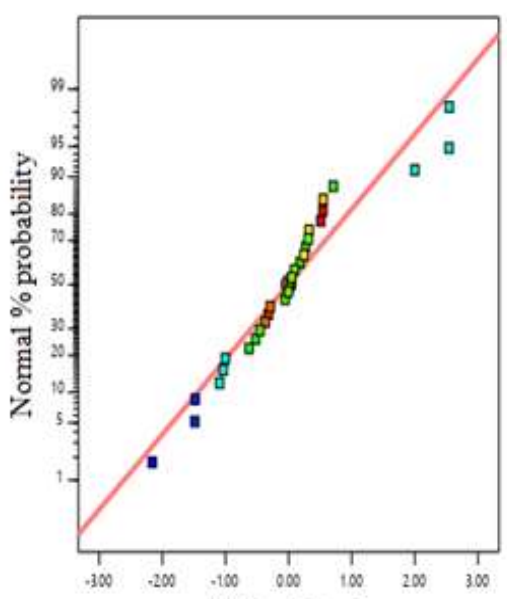

(a) Residual

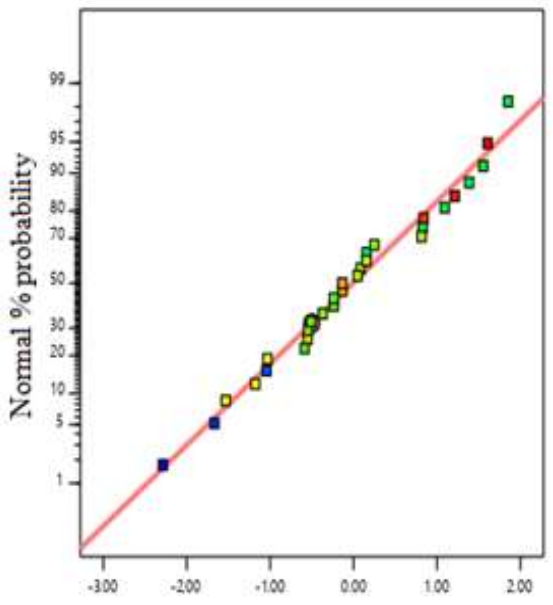

(b) Residuals

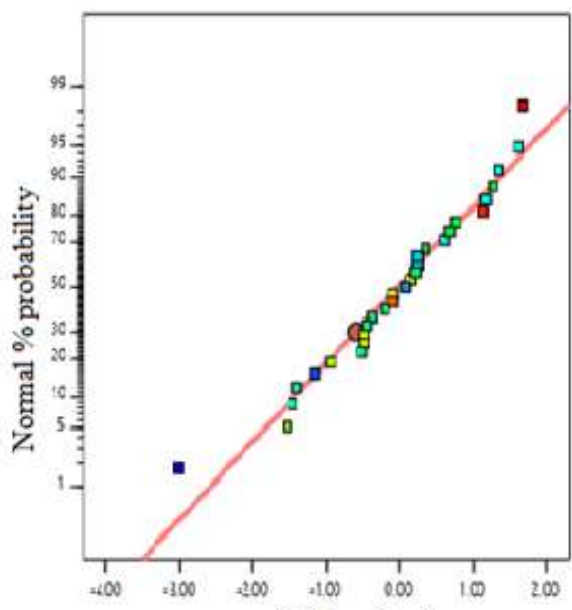

(c) Residual

Figure 8: Normal \% probability vs residual plots for (a) color strength and tensile strength in warp (b) and weft (c) direction.

\subsubsection{Effect of dye concentration on colour strength}

The dye concentration affects greatly the $\mathrm{K} / \mathrm{S}$ value of the dyed material. In the dyeing procedure, the dye concentration has an influence on both dye fixation and the color strength of the dyed fabric. Generally, as the dye concentration increases, the availability of the dye 
molecules in the dye bath also increases as a result more dye molecules can diffuse from the dye bath to the fiber [27]. Therefore, the higher the dye exhaustion causes the increase in the dye attached on and diffuses inside the fiber, the increase the dye amount inside and outside the fiber gets the advantage for fixation of a larger amount of dye on the fiber. Then the color strength of the fabric becomes increases due to the high concentration of the dye. Increasing concentration has no significant effect on tensile strength along warp and weft direction as shown in Table 3 and Figure 9. Dye concentration causes significant effects on rubbing fastness properties of both treated and untreated fabrics than other parameters.
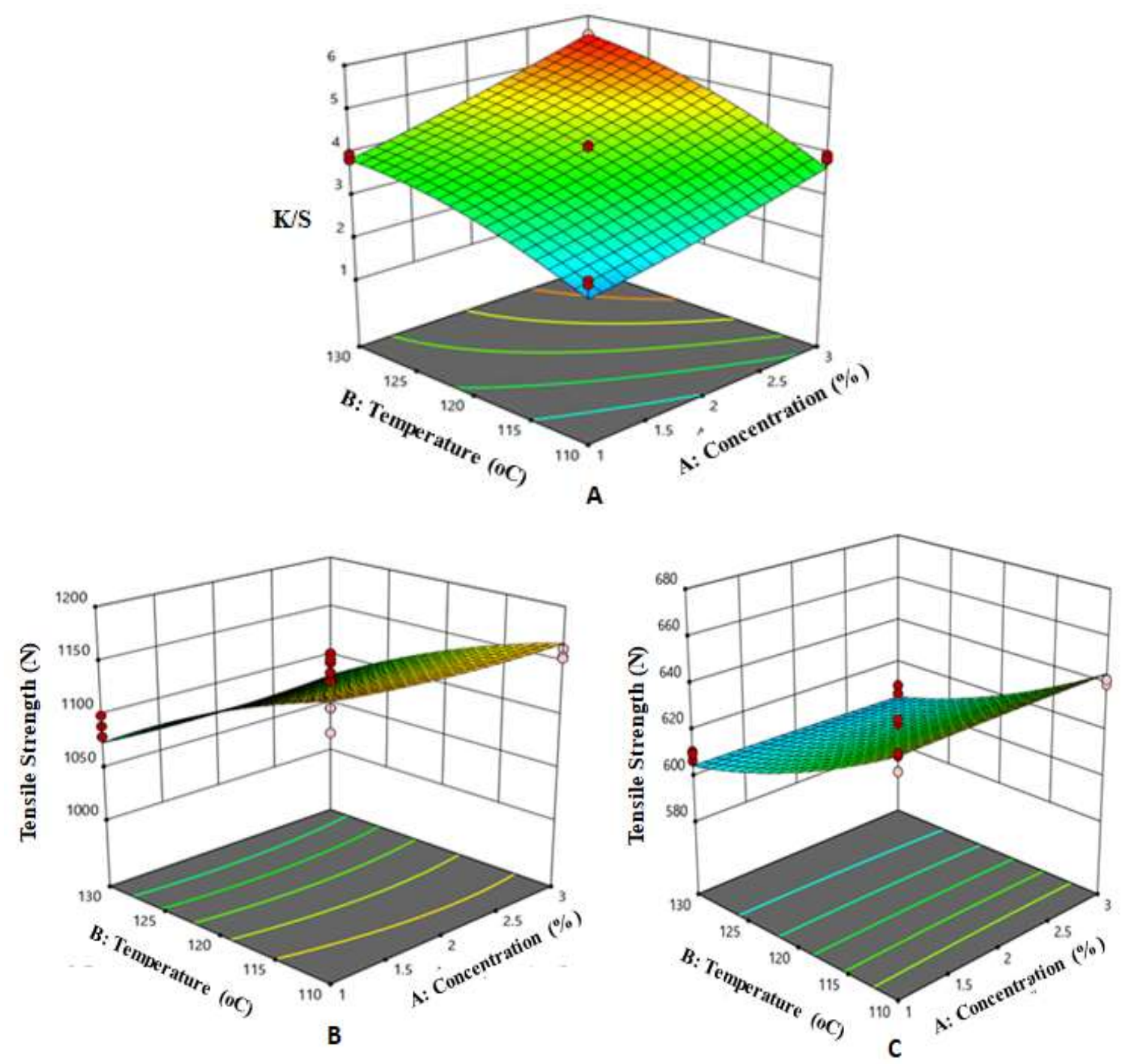

Figure 1. Optimum values for dye concentration and tensile strength in warp and weft direction. 


\subsubsection{Effect of temperature on warp and weft way tensile strength}

The effect of concentration and dyeing temperature on the responses' color strength was shown in Figure 9(a). From the result, both dye concentration and dyeing temperature are significantly increasing in color strength as it was listed in Table 3 and as increasing in dye concentration there is no significant change in tensile strength of fabric along warp and weft direction with varying the temperature. Tensile strength in the warp and weft direction was significantly affected with increasing the temperature as it was stated in Figures 9 (b) and (c) and Table 3, yet no change in strength as dye concentration was an increase. The desirability was found to be 0.7 for the preferred criteria of optimization are $1 \%$ shade at optimum dyeing temperature $120^{\circ} \mathrm{C}$ and minimum loss of tensile strength in the warp $(1143 \mathrm{~N})$ and weft $(624 \mathrm{~N})$ direction were observed. With these conditions, the tensile strength in warp and weft direction was decreased by $13 \%$ and $10 \%$ respectively compared to the control fabric samples.

\subsubsection{Effect of temperature on colour strength of modified PC blends}

As shown in Figure 9 (a) K/S value) reflects that dyeing temperature has a great impact on dye uptake for both fabrics. A dramatic change occurs in the fibre polymer system when the temperature is applied as a medium of energy. At lower temperature, 105 to $110^{\circ} \mathrm{C}$ color strength of dyed modified PC blends are low. From the result, blend composition has a higher proportion of polyester. The disperse dyeing of polyester at this temperature is difficult due to the crystal structure of polyester were not open at this temperature. It results in lower diffusion of dye into fibre structure. The color strength of modified PC blends was determined at 120,130 and $134^{\circ} \mathrm{C}$ which results in good color strength as shown in Table 3. Since the glass transition temperature of the polyester portion is reduced and its structure open to allow significant diffusion of disperse dye into fibre structure with increasing in dye fixation. Rising the temperature from 120 to $134^{\circ} \mathrm{C}$ increases the colur strength of the dyed fabric, in opposite it reduces the tensile strength of fabric along warp and weft direction. Table 3 also describes the optimized dyeing condition of modified PC blend with disperse dye in one-bath- one-step method is found at a concentration of $1 \%$ and $120^{\circ} \mathrm{C}$ of temperature with a tensile strength of $1142 \mathrm{~N}$ and $624 \mathrm{~N}$ in warp and weft direction respectively. 


\subsection{Colourfastness properties}

In general, the fastness properties of modified PC blend fabrics are dyed with disperse-yellow dye were studied with different dye concentrations (1-3\% o.w.f). Colorfastness to washing in Table 4 shows that one-bath one-step dyed PC blend with disperse dye after surface modification is approximately similar to conventional two bath-two step dyed fabric sample. The hydrophobic nature of disperse dye results in good wash fastness property of dyed fabric [28]. Overall both samples analysed are shown moderate to good fastness properties.

Table 4. Wash fastness properties of modified PC blend and conventional fabric dying.

\begin{tabular}{|l|l|l|l|l|l|l|}
\hline & \multicolumn{3}{c|}{ Single bath } & \multicolumn{3}{c|}{ Double bath } \\
\hline \multirow{2}{*}{ Shade $\%$} & Color & \multicolumn{3}{c|}{ Staining } & Color & Staining on \\
\hline 1 & Change & Cotton & Polyester & Change & Cotton & Polyester \\
\hline 2 & 4 & 4 & $4-5$ & $4-5$ & 4 & $4-5$ \\
3 & 4 & 4 & $4-5$ & $4-5$ & $4-5$ & $4-5$ \\
\hline
\end{tabular}

The result suggests a color change of one-bath one-step dyed PC blend is approximately similar to conventional two-bath two-step dyed sample. This is due to disperse dyes are hydrophobic in nature the tendency of hydrolyzed with water during washing is less and it forms strong mechanically bonding with an acetyl group and ester functional group of both polyester portion as well as acetylated cotton. Particulate disperse dye molecules accumulate at the surface of the dyed substrate because of the dye's low aqueous solubility, which impairs the brightness of the dyeing and during reductive clearing process all unreacted disperse dye removed by reductive clearing agents and only dye fiber bond formation remained and hydrophobic in nature of disperse dye results good wash fastness property.

Table 5. Rubbing fastness properties of modified PC blend and conventional fabric dying.

\begin{tabular}{|c|c|c|c|l|l|l|}
\hline & \multicolumn{3}{|c|}{ Single bath one step } & \multicolumn{3}{c|}{ Double bath } \\
\hline \multirow{2}{*}{ Dye\% } & Rubbing fastness & & \multicolumn{2}{c|}{ Rubbing fastness } & \multirow{2}{*}{ Light fastness } \\
\cline { 2 - 3 } & wet & dry & Light fastness & wet & dry & 5 \\
\hline $\mathbf{1}$ & 4 & $4-5$ & $4-5$ & 4 & 5 & $5-6$ \\
\hline $\mathbf{2}$ & 4 & 4 & 5 & $4-5$ & $4-5$ & $5-6$ \\
\hline $\mathbf{3}$ & $3-4$ & 4 & 5 & $4-5$ & $4-5$ & 5 \\
\hline
\end{tabular}


As shown in Table 5 the samples dyed in one-bath one-step disperse dye after surface modification of PC blend has good dry/wet rubbing and light fastness compared to conventional two-bath dyed samples []․

Table 6: Fastness to perspiration of modified PC blend and conventional fabric dying.

\begin{tabular}{|l|l|l|l|l|}
\hline \multirow{2}{*}{ Dye\% } & \multicolumn{2}{|l|}{ One bath dyed PC blends } & \multicolumn{2}{l|}{ Two bath dyed PC blends } \\
\hline & \multicolumn{2}{|l|}{ Perspiration fastness } & \multicolumn{2}{l|}{ Perspiration fastness } \\
\cline { 2 - 5 } & Acidic & Alkaline & Acidic & Alkaline \\
\hline $\mathbf{1}$ & 4 & 4 & 5 & 5 \\
\hline $\mathbf{2}$ & 4 & $3-4$ & 5 & $4-5$ \\
\hline $\mathbf{3}$ & $3-4$ & $3-4$ & $4-5$ & $4--5$ \\
\hline
\end{tabular}

Colorfastness to perspiration refers to the ability not to fade and not to stain when the dyed fabric is perspired. When the garments which come into contact with the body have high fastness to perspiration, it may suffer serious local discoloration. The results in Table 6 were intended to determine the resistance of color removal from dyed textile by the action of acidic and alkaline conditions. From the result, good fastness to perspiration was determined for modified PC blend under acid and alkali conditions compared to conventional two-bath dyed fabrics.

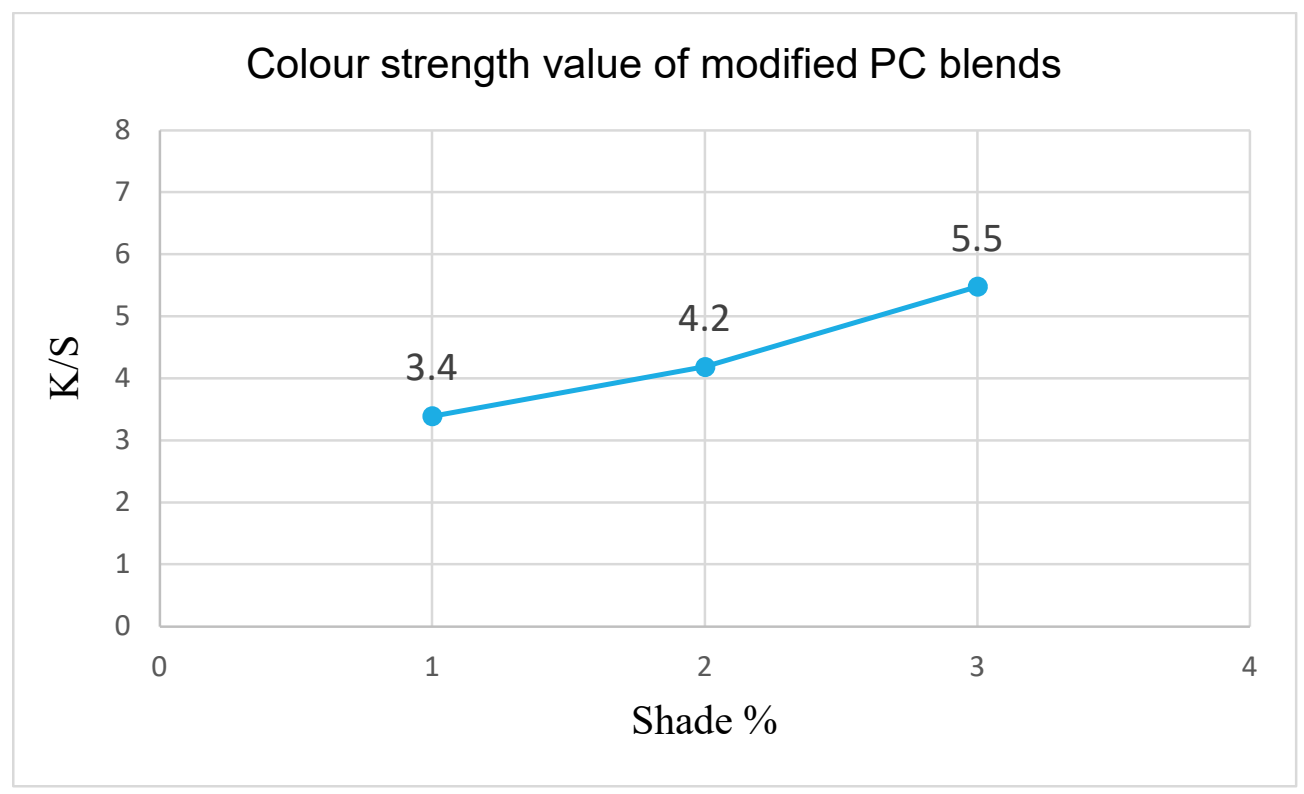

Figure 10. K/S value of modified PC blends dyed using disperse dye in one bath at $120^{\circ} \mathrm{C}$. 
Figure 10 shows the color strength of modified PC blends dyed in the one-bath one-step dyeing method. From the result obtained K/S values indicate the depth of shade reflected from the dyed fabric surface. The result represents good color strength is determined and the $\mathrm{K} / \mathrm{S}$ values were within the acceptable limit for practical dyeing. On the basis of the test results disperse dye has better affinity and dyeability with fibers ester or acetyl functional groups.

\subsection{Dyeing Mechanism}

In the mechanism of the aqueous phase transfer of disperse dyes, a greater proportion of dye is present in bulk dispersion within the dye bath, while a small amount of dye dissolves and forming an aqueous solution. Dye molecules from the aqueous solution are adsorbed at the fiber surface and then diffuse from the fiber surface to the interior of the substrate. Transfer of dye molecules from the external aqueous phase to the surface and interior of the substrate up to its saturation equilibrium corresponds to the diffusion process. Dyeing of disperse dye with hydrophobic fiber categorized under a free volume model thermodynamic dye diffusion principles, in which the diffusion of a dye molecule, which has been initially adsorbed onto a polymer chain, through a polymeric material such as fibre can only occur when the segmental mobility of the composite molecular chains is such that sufficient free volume is formed that can accommodate both the dye molecule and chain segment $[29,30]$. After surface modification of cotton parts blended polyester and cotton forms ester and acetyl hydrophobic chemical structure which conforms to free volume thermodynamic dyeing diffusion. An increase in temperature will result in a corresponding increase in the mobility of the molecular chains within the fiber, which are leads to both thermal expansion and reduced density fiber which results in an increase in dye diffusion through the open structure of the fibre. From Table 7 temperature and K/S of dyed samples were increased.

Table 7. Effect of treatment on physical properties of the fabric

\begin{tabular}{|l|l|c|c|c|c|}
\hline \multicolumn{2}{|c|}{ Property } & $\begin{array}{c}\text { Control } \\
\text { sample }\end{array}$ & $\begin{array}{c}\text { One bath dyed } \\
\text { sample }\end{array}$ & $\begin{array}{c}\text { Two bath dyed } \\
\text { sample }\end{array}$ & Change (\%) \\
\hline \multirow{2}{*}{ Tensile strength } & Warp & 1310 & 1143 & 1190 & $-13 \%$ \\
\cline { 2 - 6 } & Weft & 693 & 624 & 664 & $-10 \%$ \\
\hline \multirow{3}{*}{ Elongation (\%) } & Warp & 22 & 24 & 23 & $+8 \%$ \\
\cline { 2 - 6 } & Weft & 16 & 18 & 17 & +9 \\
\hline Tear strength(N) & Warp & 52 & 48 & 50 & $-8 \%$ \\
\cline { 2 - 6 } & Weft & 46 & 43 & 44 & $-7 \%$ \\
\hline
\end{tabular}


The tensile strength of PC blend dyed in one-bath one-step methods with disperse dye after surface modification of cotton has slightly lower than conventional two bath dyed samples. Table 7 shows the tensile strength along warp and weft directions are decreased in 13\% and 10\% respectively in one-bath one-step dyed when compared with dyed control samples. This is due to acetylation conditions and dyeing temperature reduces tensile strength of PC blends. During the acetylation process, the strength of cotton reduced due to the acetylation bath carried out in acidic media. This result shows cellulose has a low tendecy to resist both weak and strong acids. In the case of two-bath only dyeing temperature affects fabric tensile strength. From the result as temperature increased the strength of PC blend samples is reduced due to high-temperature highpressure dyeing of polyester affect the strength of cotton and acidic media of disperse dyeing of polyester portion also considered for reduction of cotton strength. The difference of strength loss between the two methods is not causing considerable loss of strength whereas acetylation methods and selected catayst made a slight effect on the tensile strenght of the fabric.

The tear strength of PC blends dyed with disperse dye in one-bath one-step methods results is almost similar compared to conventional two bath dyed samples. Table 7 shows that one-bath one-step dyed sample of modified PC blend tear strength along warp and weft direction is decreased in $8 \%$ and $7 \%$ respectively when compared with control samples because acetylation condition and dyeing temperature reduces in both tensile strength and tear strength of PC blends. From experimental results, modification PC blend can be carried out to increase the hydrophobic character of cotton by reactions that covalently bind bulky aryl residues to the fibers by acetylation process. From the result of fastness tests and abrasion resistance, we understood that good color strength was determined.

\section{Conclusion}

PC blend dyed with disperse dye in one-bath one-step dyeing methods after surface modification of cotton by acetylating methods is studied. Surface modification of cotton was carried out using fibrous acetylation methods. The result showed that the optimum value for acetylation was determined with a concentration of acetylation agent $16 \%$ and time of reaction 2.5 hours, gave a percent acetylation of 34 . The experiment result of dyeing showed that the optimum value was obtained by dye concentration above $1 \%$ at temperature $120^{\circ} \mathrm{C}$, warp tensile strength decreased by about $12 \%$ and weft tensile strength was decreased by $9 \%$ from the control half-bleached 
fabric. Therefore, techniques must be devised and studied to introduce a chemical surface modification of PC blends without significantly affecting other physical properties. The method would have a significant potential for industrial application as the one-bath one-step dyeing methods relatively safe, environmentally friendly technique, with less requirement of energy, water, time, and resource as PC blends dyed with one dye. Results of this study showed that a one-step one-bath dyed modified PC blend with disperse dye fabric presents good fastness property, abrasion resistance and tear strength value compared with conventional two-bath dyed fabric. The one-bath one-step dyeing methods of polyester/cotton blends with disperse dye also showed level dyeing having good color strength values properties and the methods offer the option of cost-effectiveness.

Authors Contribution: In this research work all authors are participate in documentation, collection of information, funding and data inputting. All authors have agreed to the published version of the manuscript.

Funding: This research was funded by Ethiopian Institute of Textile and Fashion Technology (EiTEX), Bahir Dar University, Ethiopia.

Institutional Review Board Statement: Not applicable.

Informed Consent Statement: Not applicable.

Acknowledgement: The authors would like to acknowledge the Ethiopian Institute of Textile and Fashion Technology (EiTEX) finance office.

Conflicts of Interest: The authors declare no conflict of interest.

\section{REFERENCE}

1. Rowe, H.D., Biotechnology in the textile/clothing industry-a review. Journal of Consumer Studies \& Home Economics, 1999. 23(1): p. 53-61.

2. Zou, Y., N. Reddy, and Y. Yang, Reusing polyester/cotton blend fabrics for composites. Composites Part B: Engineering, 2011. 42(4): p. 763-770.

3. Chattopadhyay, D. and T. Shaikh, Dyeing of Polyester/Cotton Blends-An Overview. MAN MADE TEXTILES IN INDIA, 2002. 45(12): p. 456-469.

4. Najafi, H., et al., One bath method dyeing of polyester/cotton blend fabric with sulphatoethylsulphonyl disperse/reactive dyes treatment by chitin biopolymer. African Journal of Biotechnology, 2009. 8(6). 
5. Prasad, R.K., M.A. Shahid, and M. Dulal, A Comparative Study Between One Bath Dyeing Method For Polyester Cotton (Pc) Blended Fabric Over Conventional Two Bath Dyeing Method. European Scientific Journal, 2015. 11(33).

6. Elsherbiny, A.S. and M. Kaukab, One-bath one-step dyeing of a polyester/cotton blend using the pad-dry-fixation process. Fibres \& Textiles in Eastern Europe, $2016(2$ (116)): p. 113--119.

7. Muralidharan, B. and S. Laya, A new approach to dyeing of 80: 20 polyester/cotton blended fabric using disperse and reactive dyes. ISRN Materials Science, 2011. 2011.

8. Meena, C., et al., One-bath dyeing process for polyester/cotton blend using physical mixtures of disperse/reactive dyes. European International Journal of Science and Technology, 2013. 2(2): p. 6-16.

9. Shahidi, S., J. Wiener, and M. Ghoranneviss, Surface modification methods for improving the dyeability of textile fabrics. Eco-Friendly Textile Dyeing and Finishing, 2013: p. 34-50.

10. Tausif, M., et al., A comparative study of mechanical and comfort properties of bamboo viscose as an eco-friendly alternative to conventional cotton fibre in polyester blended knitted fabrics. Journal of Cleaner Production, 2015. 89: p. 110-115.

11. Baiardo, M., et al., Surface chemical modification of natural cellulose fibers. Journal of Applied Polymer Science, 2002. 83(1): p. 38-45.

12. Lisperguer, J., et al., The effect of wood acetylation on thermal behavior of woodpolystyrene composites. Journal of the Chilean Chemical Society, 2007. 52(1): p. 10731075.

13. Ashori, A., et al., Solvent-free acetylation of cellulose nanofibers for improving compatibility and dispersion. Carbohydrate polymers, 2014. 102: p. 369-375.

14. Hill, C., et al., Kinetic and mechanistic aspects of the acetylation of wood with acetic anhydride. Holzforschung-International Journal of the Biology, Chemistry, Physics and Technology of Wood, 1998. 52(6): p. 623-629.

15. Saha, P., et al., A brief review on the chemical modifications of lignocellulosic fibers for durable engineering composites. Polymer Bulletin, 2016. 73(2): p. 587-620.

16. El Nemr, A., S. Ragab, and A. El Sikaily, Testing zinc chloride as a new catalyst for direct synthesis of cellulose di-and tri-acetate in a solvent free system under microwave irradiation. Carbohydrate polymers, 2016. 151: p. 1058-1067.

17. Anbu, N., et al., Acetylation of alcohols, amines, phenols, thiols under catalyst and solvent-free conditions. Chemistry, 2019. 1(1): p. 69-79. 
18. Bello, A., et al., Acetylation of cotton stalk for cellulose acetate production. American Scientific Research Journal for Engineering, Technology, and Sciences (ASRJETS), 2016. 15(1): p. 137-150.

19. Mantanis, G., R. Young, and R. Rowell, Swelling of compressed cellulose fiber webs in organic liquids. Cellulose, 1995. 2(1): p. 1-22.

20. Frisoni, G., et al., Natural cellulose fibers: heterogeneous acetylation kinetics and biodegradation behavior. Biomacromolecules, 2001. 2(2): p. 476-482.

21. El Nemr, A., et al., Synthesis of cellulose triacetate from cotton cellulose by using NIS as a catalyst under mild reaction conditions. Carbohydrate polymers, 2015. 130: p. 41-48.

22. Stenstad, P., et al., Chemical surface modifications of microfibrillated cellulose. Cellulose, 2008. 15(1): p. 35-45.

23. Zhuang, J., et al., Observation of Potential Contaminants in Processed Biomass Using Fourier Transform Infrared Spectroscopy. Applied Sciences, 2020. 10(12): p. 4345.

24. Hussain, U., et al., Comfort and mechanical properties of polyester/bamboo and polyester/cotton blended knitted fabric. Journal of Engineered Fibers and Fabrics, 2015. 10(2): p. 155892501501000207.

25. Yan, L., B. Kasal, and L. Huang, A review of recent research on the use of cellulosic fibres, their fibre fabric reinforced cementitious, geo-polymer and polymer composites in civil engineering. Composites Part B: Engineering, 2016. 92: p. 94-132.

26. Tsuji, W., R. Kitamaru, and M. Imai, Studies on the Fibrous Acetylation of Cotton.(II): Properties of the Acetylated Cotton Prepared by the Liquid Phase Method (Special Issue on Polymer Chemistry, II). 1965.

27. Shahid, M.A., et al., Effect of different dyeing parameters on color strength \& fastness properties of cotton-elastane (ce) and lyocell-elastane (le) knit fabric. International Journal of Textile Science, 2016. 5(1): p. 1-7.

28. Micheal, M., F. Tera, and S. Ibrahim, Effect of chemical modification of cotton fabrics on dyeing properties. Journal of applied polymer science, 2002. 85(9): p. 1897-1903.

29. McKenna, G.B., Glass formation and glassy behavior. Pergamon Press plc, Comprehensive Polymer Science: the Synthesis, Characterization, Reactions \& Applications of Polymers., 1989. 2: p. 311-362.

30. Padbury, R.P., Bulk Property Modification of Fiber Forming Polymers using Vapor Phase Techniques. 2014. 